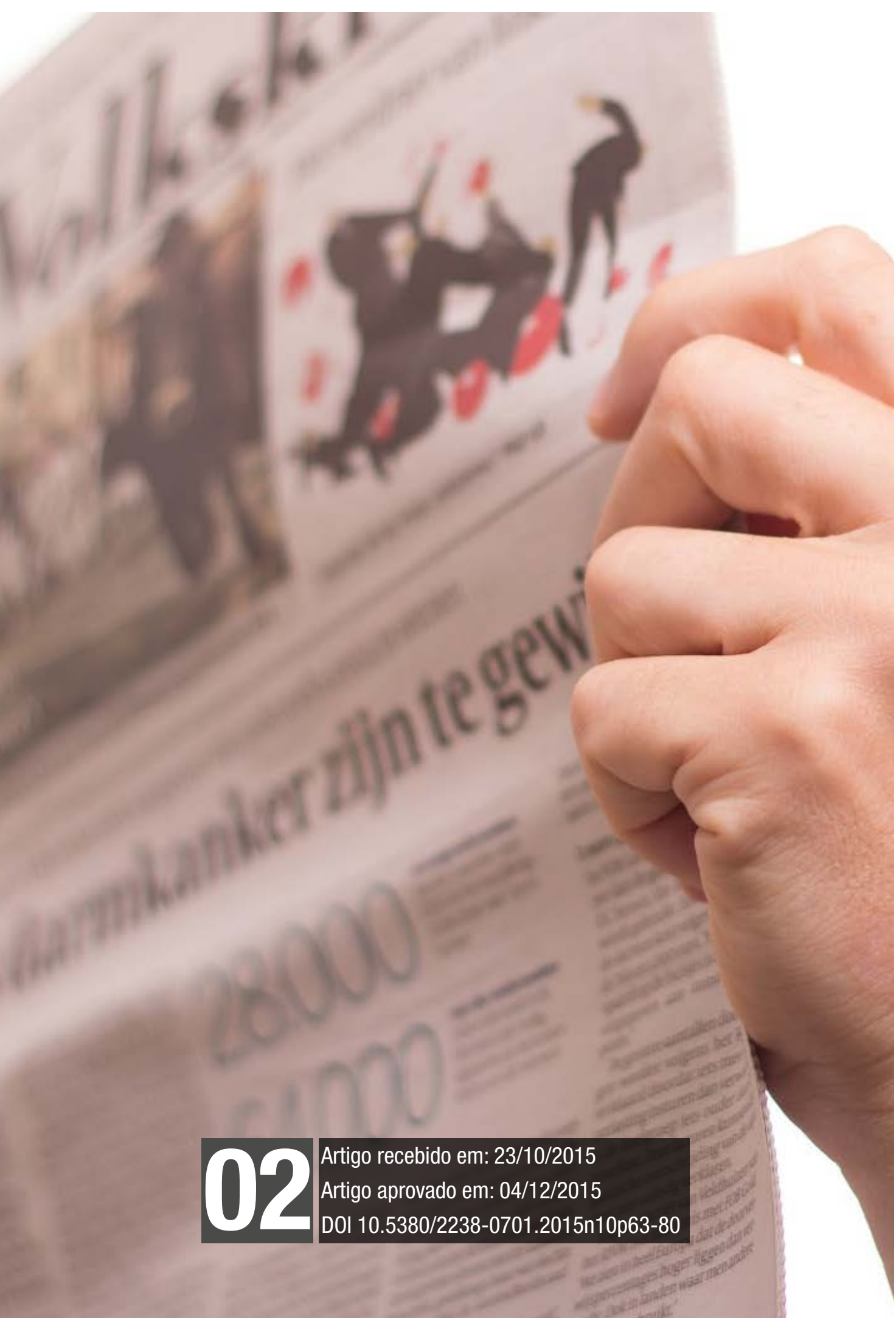


AÇÃO MIDIÁTICA, n. 10. Ju/Dez. 2015. Curitiba. PPGCOM-UFPR. ISSN 2238-0701 


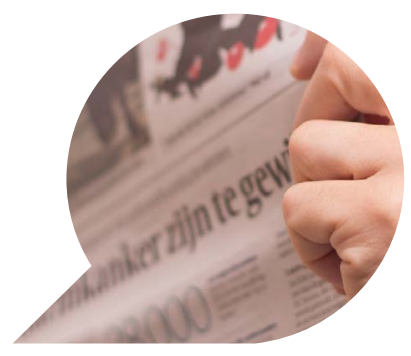

\title{
Le discours « positionnel » du Huffington Post: permanence et mobilité d'une narration stratégique
}

\author{
O discurso posicional do Huffington Post: \\ permanência e mobilidade de uma narrativa estratégica
}

\author{
The positionnal discourse of the Huffington Post: \\ a narrative strategiccaly mobile yet invariable
}

\begin{tabular}{c} 
ARNAUD NOBLET $^{*}$ \\
\hline RAYYA ROUMANOS $^{* *}$ \\
\hline
\end{tabular}

Résumé: Cet article examine lévolution de la communication institutionnelle du Huffington Post depuis son lancement en 2005. Il étudie la stratégie de positionnement du média sur une période de dix ans ponctuée de bouleversements considérables tant au niveau la structure du pure player que de son contenu. À travers l'examen de son discours positionnel, assumé essentiellement par sa fondatrice et actuelle directrice générale Arianna Huffington, il met en évidence la cohabitation de deux postures de prime abord contradictoires : l'une insistant

\footnotetext{
* Maître de conférences en sciences de l'information et de la communication, diplômé de l'École Supérieure de Journalisme de lille (USJ), il est actuellement responsable du pôle journalisme de l'Institut de la Communication de l'université Lumière Lyon 2. a.noblet@univ-lyon2.fr

${ }^{*}$ Maître de conférence à l'Institut de journalisme de l'université de Bordeaux Montaigne (IJBA) et membre du laboratoire MICA EA 4426. rayroumanos@gmail.com
} 
sur la stabilité du média, bâtis sur un socle de pratiques et de valeurs immuables et l'autre exaltant les vertus du changement quand bien même ce dernier bouscule ces mêmes pratiques et valeurs. Si le discours reste opérant, c'est grâce au déplacement de son objet principal du média en lui-même (son mode de fonctionnement) vers le rôle social du Journalisme, à travers une narration idéologique axée sur l'exigence de transparence, de vérité et de participation.

Mots clés: Huffinton Post; Journalisme en ligne; Discours positionnel.

Resumo: Este artigo examina a evolução da comunicação institucional do Huffington Post desde seu lançamento em 2005. Ele lança um olhar sobre a estratégia de posicionamento desta mídia em um período de dez anos, pontuado por consideráveis mudanças estruturais e editoriais. Por meio da análise de seu discurso institucional, apresentado principalmente por sua fundadora e atual diretora, Arianna Huffington, evidencia a associação de duas posturas à primeira vista contraditórias: uma que representa a estabilidade de um certo número de práticas e valores jornalísticos imutávies, e outra que exalta a importância da mudança que causa perturbações a estas mesmas práticas e valores. Se o discurso permanece efetivo é graças ao deslocamento de seu objeto principal, a mídia em si mesma (seu modo operacional), para os papeis sociais do Jornalismo, através de uma narrativa ideológica baseada em uma demanda pública por transparência, verdade e participação.

Palavras-chave: Huffington Post; Jornalismo Digital; Discurso institucional.

Abstract: This article examines the evolution of the institutional discourse of the Huffington Post since 2005. It looks at its positioning strategy over the last 10 years in the light of the considerable structural and editorial changes that this net-native went through. The analysis of its corporate narrative, presented mainly by its founder and current director Arianna Huffington, 
highlights the association of two seemingly contradictory postures: one that values the stability of a select number of practices and values and another that acknowledges the importance of change, notwithstanding its faculty to disturb these same standards and goals. If this discourse remains effective it is because of its ability to deviate its main object from the media in itself (its operating mode) to the social roles of Journalism, through an ideological narrative based on the public's demand for transparency, accuracy and participation.

Keywords: Huffington Post; Online Journalism; Positional discourse. 


\section{Introduction}

Le Huffington Post, lancé aux États-Unis en mai 2005, a aujourd'hui 10 ans. Né la même année que YouTube', il a reproduit le succès de cette plateforme en se transformant en média mainstream tirant profit des larges potentialités du web. D’ailleurs, il a lui-même été, à ses débuts, un agrégateur de contenu participatif avant de se convertir en entreprise de presse multi-supports, multi-plateformes et internationale.

Son histoire, ponctuée de plusieurs moments forts, dont son rachat par AOL et son ouverture à l'international en 2011, est, en fait, celle d'une expansion continuelle censée surpasser le « dilemme de l'innovateur $»^{2}$, selon les termes de sa fondatrice et actuelle directrice générale, Arianna Huffington.

Sa communication institutionnelle insiste sur cette stratégie commerciale qui consiste à " évoluer», à " innover » et à "se réinventer» continuellement pour rester compétitif. Pourtant, malgré ces changements, dont l'effet le plus important reste le glissement d'une logique de compilation arbitraire de billets d'humeur vers une politique d'amplification d'un contenu journalistique ordonné, orienté et viral, son discours positionnel a conservé une certaine stabilité en mettant en avant la principale " mission » du média : délivrer une information "participative », " authentique » et " instantanée ». ${ }^{3}$

Lobjet de notre texte est d'étudier cette apparente contradiction pour tenter de déterminer la place qu'occupe (ou cherche à occuper) le Huffington Post dans le système médiatique qui le voit naitre et s'accroitre.

Dans cette perspective, nous essayerons de répondre à trois questions complémentaires : quelles sont les caractéristiques du discours

\footnotetext{
1 YouTube a été lancé en février 2005

2 Arianna Huffington emprunte cette expression à Clayton Christensen (The Innovator's Dilemma: When New Technologies Cause Great Firms to Failla. Boston : Harvard Business Press, 1997). Expliquant les raisons du succès de son média dans un éditorial de septembre 2014 consacré au dépassement de la barre des 100 millions de visiteurs uniques, elle dit : "at every step along the way, we refused to fall prey to the innovator's dilemma by constantly evolving, innovating and reinventing vital aspects of how we put together The Huffington Post while remaining true to the core values that made HuffPost HuffPost", HUFFINGTON Arianna, " 100 Million Thank-Yous to HuffPosters Around the World", huffingtonpost.com, September 15, 2014, available at: <http:// www.huffingtonpost.com/arianna-huffington/100-million-thank-yous-to-huffposters-aroundthe-world_b_5822998.html.

3 HUFFINGTON Arianna, "Celebrating HuffPost's First 10 Years by Looking to the Next 10", huffingtonpost.com, May 6, 2015, available at: <http://www.huffingtonpost.com/ariannahuffington/huffpost-10-years_b_7225620.html>
} 
positionnel du Huffington Post? Que dévoile-t-il sur la nature du média et sur sa place dans l'espace médiatique qui l'accueille? Comment agit-il sur les débats qui animent la profession autour de problématiques telles que le rôle du journalisme à l'heure du numérique ou les évolutions des rapports entre médias, producteurs et usagers?

Pour répondre à ces questions, nous nous basons sur un ensemble d'éditoriaux et articles de presse regroupant les déclarations de la fondatrice du média, Arianna Huffington, ainsi que de ses collaborateurs lors d'évènements significatifs de l'histoire du Huffington Post, comme sa mise en ligne en mai 2005, le lancement de son projet de journalisme politique participatif «Off the Bus» en avril 2008, son rachat par AOL en février 2011, le prix Pulitzer remporté en 2012 ou encore le lancement de ses différentes éditions internationales. Étalées sur dix ans, ces annonces permettent de saisir non seulement l'évolution de la stratégie de positionnement du Huffington Post, mais aussi, en les confrontant aux évolutions du secteur de la presse, les changements qui s'opèrent dans le journalisme à des échelles aussi bien locales que globales.

Notre texte sera divisé en trois parties : la première explorera lobjet central du discours positionnel du Huffington Post qui se révèle être le journalisme envisagé dans sa dimension politique plutôt que le média lui-même. Puis dans la seconde partie, nous nous attarderons sur les permanences de la narration institutionnelle que nous confronterons; dans la troisième, à la stratégie de communication mobile adoptée par le média.

\section{Un discours positionnel sur le journalisme plutôt que le média}

Notons de prime abord que nous employons le terme « discours positionnel » dans le sens d'une narration performative produite par les représentants du Huffington Post et destinée à désigner la place qu'occupe (ou cherche à occuper) le média dans le système. En tant qu'acte central d'une stratégie de communication institutionnelle plus globale, il est censé participé à la promotion de l'image du Huffington Post et à la fixation de son identité dans un contexte très concurrentiel. Ce discours n'est pas figé, mais évolue dans le temps pour s'adapter à l'environnement dans lequel le média opère. Il n'est pas non plus lisse, mais comprend des exagérations, des non-dits, 
des contradictions, etc. qui témoignent des agitations qui traversent l'espace médiatique qui l'accueille.

L'analyse de notre corpus de recherche composé d'un ensemble de déclarations étalées sur une période de dix ans montre que l'une des particularités de cet acte discursif, surtout durant les premières années d'existence du média, est d'être un discours engagé construit en deux axes : le premier consiste à montrer du doigt les défaillances de la presse américaine et internationale et le second à présenter le pure player comme une source d'information alternative.

Pour exposer la mission du Huffington Post et son « contrat de lecture »(CHARAUDEAU, 1997, p.67), Arianna Huffington et ses collaborateurs opposent, dès 2005, leur projet à celui des MSM (comprendre MainStream Media ${ }^{4}$ ou médias grand public), qu'ils rendent directement et exclusivement responsables de la crise du journalisme au $21 \mathrm{e}$ siècle ${ }^{5}$. Leurs éditoriaux des premières années s'attaquent régulièrement à cette catégorie de supports journalistiques qui est composée, selon eux, des médias partisans dont les journalistes, souvent complaisants avec certains groupes sociaux ont, en plus, un regard élitiste sur l'actualité et manquent de créativité au niveau du choix des sujets et de leur traitement. Ils leur reprochent surtout de ne plus respecter la mission première du journalisme qui consiste à fournir aux citoyens les informations dont ils ont besoin pour prendre les meilleures décisions possible au sujet de leurs vies, de leurs communautés, de leurs sociétés et de leurs gouvernements.

A contrario, ils assurent que le Huffington Post offre, de par sa nature et sa structure, une alternative destinée à pallier les défauts des médias mainstream, tout en réenchantant un secteur de la presse moribond. Ils expliquent que leur média est indépendant, multi-voix, multi-médias et multi-plateformes. C'est une plateforme participative qui favorise les débats publics et encourage les conversations citoyennes. C'est aussi un réceptacle de narrations intimes et authentiques, libérées des codes classiques de l'écriture journalistique. $\mathrm{Ni}$

4 Cette abréviation a été forgée aux États-Unis par des blogueurs et comporte, de ce fait, un caractère péjoratif http://articles.philly.com/2005-05-16/news/25441772_1_instapunditcandidate-blogs-glenn-harlan-reynolds>

5 L'appellation Huffington Post témoigne de cette stratégie d'opposition. Dans une interview accordée à Newsweek au lendemain du lancement du site, Arianna Huffington explique que le nom de son média est « un jeu de mots qui rappelle le titre de média mainstream comme le Washington post. Sauf que nous, nous 'postons' des billets. Il y a une forme d'irrévérence dans ce que nous faisons, nous ne nous prenons pas trop au sérieux, d’où notre slogan : « un média qui délivre des informations et des opinions depuis le 9 mai 2005 » 
partisan ni surplombant et refusant de s'engager dans le journalisme « embarqué » (entendu au sens de connivence entre les hommes politiques et les journalistes) le Huffington Post est un média viral qui associe les fondamentaux du journalisme (la vérification des faits, la poursuite de la vérité, l'exactitude et l'équité) aux apports avantageux des nouvelles technologies (l'immédiateté, la transparence et l'engagement au niveau de la couverture des faits).

Dans ces énoncés, le nombre d'indications sur la ligne éditoriale du Huffington Post ou sur son mode de fonctionnement est inversement proportionnel aux allusions au rôle social que souhaite endosser le média. En d'autres termes, le discours positionnel du HuffPo serait essentiellement une narration sur le journalisme et sa fonction sociale, avant dêtre un discours sur le média. Sa communication institutionnelle se place, de ce fait, du côté de l'énonciation de l'utilité sociale d'une telle entreprise qui répond à trois types d'exigences identifiées par les gestionnaires du groupe : exigences de transparence, de vérité et de participation.

À travers son discours, le Huffington Post se désigne comme un " médiateur » entre les usagers et l'information opérant dans une double optique pragmatique et politique. D'un point vue pragmatique, il se présente comme un intermédiaire « neutre » qui récupère et publie des articles sans leur faire subir une quelconque censure stylistique ou idéologique, et d'un point de vue politique, il se positionne comme un facilitateur du processus démocratique par la réinscription des citoyens au centre des débats publics.

L'une comme l'autre de ces postures est évidemment abusive, mais exposer leur défaillance dépasse les cadres de cette argumentation. Nous nous contenterons de souligner ici l'instance des promoteurs du site sur la dimension politique du journalisme qui devient le vecteur principal du discours positionnel du Huffington Post.

Son autre particularité est de conserver, malgré les évoluons du site, une certaine stabilité.

\section{Une narration stable en dépit des changements}

De fait, l'étude des déclarations d'Arianna et de ses collaborateurs montre clairement que la communication institutionnelle du Huffington Post se caractérise par une forme de constance, puisqu'en 
dehors de l'ampleur des attaques contre les MSM qui ont effectivement diminué à partir du moment où le Huffington Post a rejoint ce groupe à la fin des années 2000, les autres critères de description du média sont, eux, restés stables, malgré les nombreux bouleversements qu'a connus le Huffington Post au fil des ans.

Au niveau de sa nature d'abord, le média a subi plusieurs transformations puisqu'il est passé d'un agrégateur de billets d'opinion à un média d'information généraliste. En 2005, il a été présenté à la presse comme " un blog innovant qui permettra aux esprits les plus créatifs des USA ${ }^{6}$ de peser dans le débat public sur différents sujets ${ }^{7}$. Trois ans plus tard, le « super blog d'Arianna $"{ }^{8}$ essentiellement alimenté par des billets rédigés par des célébrités hollywoodiennes et des représentants démocrates, introduit une nouvelle offre dédiée à la couverture des élections présidentielles américaines. Intitulée " off the bus », cette édition spéciale est en fait un projet de journalisme participatif qui propulsera le Huffington Post dans l'arène des grands médias d'information et marquera le début de sa réorientation vers le journalisme d'information (par opposition au journalisme d'opinion). Une tendance confortée par un ensemble d'actions dont l'accroissement progressif de l'équipe éditoriale (qui compte aujourd'hui 575 journalistes), la multiplication de verticales aux titres plutôt classiques (Front Page, Politics, Business, entertainement, Tech, Media), l'investissement dans le journalisme hyper-local à travers la reprise des sites Patch.com, propriétés du groupe AOL qui rachète le Huffington Post en février 2011, l'obtention du prix Pulitzer en avril 2012, le lancement du HuffPo Live, chaine d'information en continu et en ligne en aout 2012 ou encore la création d'un WordPost dédié à l'actualité internationale, en janvier 2014.

$\mathrm{Au}$ niveau de sa structure, le Huffington Post passe d'un site unique comportant un nombre réduit de rubriques, à un média éclaté fédérant plusieurs verticales et un nombre toujours croissant d'éditions. Progressivement, ses équipes diversifient les plateformes

6 Il s'agit en fait des personnes inscrites dans le carnet d'adresses très sélectif d'Arianna Huffington dont les contributions, plus ou moins régulières, plus ou moins pertinentes, devaient être complétées par des brèves d'actualité et des liens vers des articles publiés en ligne par des médias américains conventionnels.

7 Éditorial inaugural rédigé par Arianna Huffington et publié en ligne le 5 mai 2005 sur le huffingtonpost.com.

8 Terme railleur adopté par la presse américaine au moment du lancement du Huffington Post aux USA. 
et introduisent nouveaux services dont un celui dédié à la gestion des " native ads» (contenus sponsorisés) : le HuffPost Partner studio". Parallèlement à cela, le groupe a mis en place une stratégie de développement sur les réseaux sociaux destinée à intensifier la présence du média sur Twitter et Facebook principalement. Cette expansion impressionnante est le résultat direct du rachat du pure player en 2011 par AOL, le géant américain des services internet, qui mettra à la disposition d'Arianna, nommée directrice générale du groupe Huffington, les infrastructures et les fonds nécessaires à la conquête des villes américaines puis du monde. C’est alors que le caractère « omnibus » du média se révèle fondamental dans la machinerie HuffPo dont le succès dépend aujourd'hui de la captation du plus grand nombre d'usagers.

D’autre part, si le modèle économique du pure player reste inchangé et repose principalement sur les revenus publicitaires, l'échelle a, elle, été complètement modifiée par le rachat du média par AOL et par son ouverture à l'internationale. Depuis, le média a considérablement élargi son département de publicité et a diversifié son offre pour augmenter ses revenus. Il a également développé une stratégie axée sur la viralité du web en créant un service spécialisé dans le repérage et la publication de contenu à grand potentiel de diffusion, chapeauté par un "Senior Editor of Viral content and Strategy ».

Enfin, d'un point de vue éditorial, le média qui a commencé comme une plateforme de diffusion d'un contenu " progressiste » alimenté par des collaborateurs issus principalement des courants libé$\operatorname{raux}^{10}$, est devenu une plateforme beaucoup plus ouverte qui publie des informations chaudes, des commentaires de tout bord sur l'actualité, et des informations divertissantes étalées sur plus de 40 verticales. Cette tendance à l'ouverture a du reste été renforcée par la création d'éditions internationales, le plus souvent en partenariat avec des médias établis, parfois même conservateurs, comme c'est le cas au Brésil.

Malgré ces multiples changements, les termes employés par les gestionnaires du Huffington Post pour définir leur média sont restés stables. La narration déployée suit un fil rouge immuable (insistance

9 Le Huffington Post propose à la fois un service de gestion du contenu sponsorisé et une verticale dédié à ce genre de publicité cachée : <http://www.huffingtonpost.com/news/sponsoredcontent/>

10 “Greek-born socialite Huffington joins battle of the blogs",AFP, May 9, 2005, available at: <http:// www.smh.com.au/news/Breaking/Socialite-joins-the-battle-of-the-blogs/2005/05/10/ 1115584940440.html> 
sur le caractère participatif du média), traversé par des variations continuelles, mais périphériques. Arianna Huffington et ses collaborateurs ne réinventent pas la marque à chaque altération, quand bien même les réformes subies en dix ans sont, en réalité, structurelles, mais présentent les innovations comme autant de colonnes qui viennent s'ajouter à un édifice solide appelé à s'agrandir. C'est ainsi que pour annoncer le lancement d'une nouvelle verticale ou d'une énième édition, Arianna Huffington emploie des termes qui évoquent la permanence et la continuité comme les " piliers " "l'ADN » ou la " signature " du Huffington Post. C'est pour cette raison également que lon retrouve les mêmes éléments de langage dans la plupart des éditoriaux consacrés à la communication institutionnelle.

Pour autant, cette stabilité apparente n'est pas synonyme de clarté. Quand il s'agit de définir la nature exacte de ce média, les usagers sont confrontés à une multitude d'informations contradictoires qui rendent difficile ce travail de désignation. Le Huffington Post est-il un « super blog»? Un agrégateur de contenu? Un producteur de contenu original ? Une plateforme de discussion? Un journal en ligne? etc.

Cette confusion transparait également dans les commentaires des spécialistes des médias qui parlent tantôt d'un média mainstream soumis aux mêmes exigences professionnelles que ses concurrents, ou d'un média alternatif qui crée ses propres codes. Qu'ils s'extasient devant sa réussite commerciale ou qu'ils l'accablent de reproches ${ }^{11}$, ils privilégient l'une ou l'autre de ces deux définitions. À tel point que Nate Silver se sent obligé d'expliquer en 2011, à la suite de la polémique sur le modèle de journalisme contributif gratuit proposé par le Huffington Post (la crise \#HuffPuff) ${ }^{12}$, que les détracteurs du média ont, en fait, une mauvaise conception de la nature de ce dernier. Selon lui, il ne s'agit pas d'une entreprise éditoriale classique, mais plutôt une entreprise technologique, comme Facebook, dont le succès dépend exclusivement des techniques de captation de l'audience et non du contenu, qu'il soit ou non rémunéré. ${ }^{13}$

11 Les détracteurs du Huffington Post lui reprochent essentiellement de dévaloriser le journalisme (à travers son modèle qui gomme les frontières entre information, divertissement et publicité) et de précariser les journalistes (à travers sa politique d'agrégation de contenu et de non-rémunération des collaborateurs).

12 RUSHKOFF Douglas, Huffington Post and AOL: the end of Web 2.0, The Guardian, February 7, 2011, available at: <http://www.theguardian.com/commentisfree/cifamerica/2011/feb/09/ arianna-huffington-aol-douglas-rushkoff>

13 SILVER Nate, "The Economics of Blogging and The Huffington Post", The New York Times, February 12, 2011, available at: <http://fivethirtyeight.blogs.nytimes.com/2011/02/12/the- 


\section{Une stratégie de communication « mobile»}

Où se positionne le Huffington Post sur la carte virtuelle des médias en ligne? Sa communication institutionnelle apporte-t-elle des réponses claires à cette question?

Si lon se réfère au schéma imaginé par Marc Deuze pour cartographier les sites d'information en ligne du point de vue de l'énonciation (DEUZE, 2003, p.205), nous constatons que le Huffington Post oscille le long de deux continuums : le domaine de la communication participative (le degré de contrôle éditorial) et celui de la connectivité (le degré de participation).

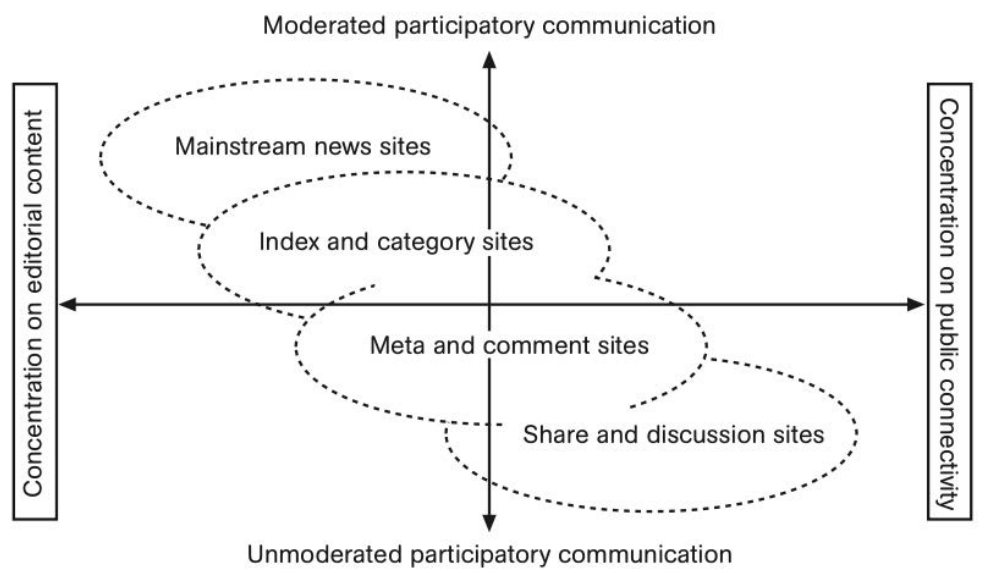

Figure 1: A typology of online journalism, DEUZE, 2003, p. 205

Ces deux axes perpendiculaires ont pour extrémités, sur le plan vertical un contenu subissant la modération des gestionnaires des sites v/s un contenu libre de tout contrôle (Participatory Communication Domain) et sur le plan horizontal un contenu éditorial produit par des professionnels v/s un contenu proposé par le public (Content-Connectiviy Domain).

Entre les quatre on retrouve dans le coin supérieur gauche les médias mainstream qui associent le contenu éditorial au contrôle de ce même contenu et dans le coin inférieur opposé les forums de discussion qui réunissent le contenu libre produit exclusivement par les usagers. Entre ces deux extrémités, sur un plan diagonal, sont placés les agrégateurs de contenu et les sites de métadiscours et de commentaires. 
Le Huffington Post a débuté comme une plateforme ouverte accueillant des billets non journalistiques subissant un contrôle éditorial très lâche tout en se présentant (se positionnant) comme un média d'information alternatif au MSM. Puis, à partir des premiers succès éditoriaux, il est devenu un MSM mettant l'accent le caractère " dialogique de sa production journalistique » (MARTIKAINEN, 2000).

L'habilité de ses communicants a consisté à éviter l'enfermement définitif du média dans l'une ou l'autre de ces catégories pour tirer profit des atouts que chacune présente. Grâce à cette stratégie de communication que l'on peut qualifier de "mobile », en reprenant une expression forgée par François Jost dans le cadre de l'analyse des genres télévisuels, ${ }^{14}$ ils se sont offert le loisir de se revendiquer de tel ou tel modèle, selon le contexte de lénonciation.

En 2005, Arianna Huffington insiste sur le caractère " intime» et « libre » des contributions, qui relève d'une forme de journalisme d'opinion plutôt que d'un journalisme d'information. ${ }^{15}$

En 2008, elle souligne le caractère journalistique du projet « Off the bus » qui s'appuie sur les contributions d'internautes chapeautés par des journalistes professionnels. ${ }^{16}$

En 2010, elle insiste sur l'importance de la participation de contributeurs non professionnels en annonçant le lancement de l'outil Live Blogging.

En février 2011, elle rappelle que son média est essentiellement un lieu de conversation multi-voix et continue, surtout depuis la fusion du groupe avec les sites de journalisme hyperlocal Patch.com devenus «Local Voices » (voix locales). ${ }^{17}$

En mai 2011, date de lancement du Huffington Post Canada, puis à chaque lancement d'une nouvelle édition internationale, elle met

14 François Jost qualifie de mobile une stratégie de communication qui change d'appui en fonction du contexte d'énonciation. Analysant la communication autour de l'émission de téléréalité Loft Story, il constate l'habilité de ses promoteurs qui se sont "réservé cet éventail de possible pour 'vendre' le programme, ce qui leur a permis d'adopter une stratégie de communication mobile. Quand une bataille semblait perdue sur l'une de ses trois positions [la mise en avant d'un des trois modes ludique, fictif et authentifiant], il suffisait de l'abandonner brutalement au profit d'une autre et de laisser son interlocuteur face à ses contradictions ». (JOST F., 2009, p.111)

15 SEELYE Katharine Q., "A Boldface Name Invites Others to Blog With Her", New York Times, April 25, 2005, available at: <http://www.nytimes.com/2005/04/25/technology/a-boldfacename-invites-others-to-blog-with-her.html?_r=0>

16 HUFFINGTON Arianna, "OffTheBus: HuffPost's Citizen Journalism Project Gets A Name, and Gets Rolling", huffingtonpost.com, April 10, 2008, available at: <http://www.huffingtonpost. com/arianna-huffington/offthebus-huffposts-citiz_b_52712.html>

17 HUFFINGTON Arianna, "Local Voices: Hyperlocal Blogging Comes to Patch", May 4, 2011, huffingtonpost.com, available at: <http://www.huffingtonpost.com/arianna-huffington/localvoices-hyperlocal-b_b_857782.html> 
en avant les qualités journalistiques de son média et introduit dans son discours la formule suivante: les éditions nationales conservent la signature du Huffpo qui combine articles d'information, d'opinion, de divertissement, et un aspect communautaire, le tout adapté aux attentes du lectorat local. ${ }^{18}$

En avril 2012, à la suite de l'obtention du prix Pulitzer, la fondatrice dit tenir enfin la preuve qu'un journalisme de qualité peut être pratiqué sur n'importe quel type de plateforme et non seulement dans les médias traditionnels. ${ }^{19}$

En aout 2012, lors de la création de la chaine d'information en continu HuffPost Live, elle lance un appel à contribution dans un éditorial titré « We need you ! $»^{20}$ (On a besoin de vous) au moment où le directeur éditorial de la chaine Roy Sekoff insiste sur le caractère innovant du média qui s'appuie sur des « citoyens souhaitant s'engager dans le débat public ». ${ }^{21}$ La critique quant à elle parle d'une plateforme basée sur un modèle classique de journalisme télévisuel dans lequel les filtres techniques restent importants malgré une certaine liberté éditoriale et stylistique. ${ }^{22}$ Mêmes remarques lors du lancement du magazine pour Ipad intitulé sobrement Huffington en 2012.23

Enfin, en janvier 2014, pour le lancement du WordPost, Arianna Huffington parle d'une plateforme qui sorganise autour des contributions de chercheurs de l'Institut Berggruen, de correspondants du site et de blogueurs anonymes, de manière à « combiner les avantages du journalisme traditionnel à ceux issus des nouvelles technologies $» .{ }^{24}$

18 HUFFINGTON Arianna, "HuffPost Goes International: Introducing HuffPost Canada", huffingtonpost.com ,May 26, 2011, available at: <http://www.huffingtonpost.ca/ariannahuffington/huffpost-canada_b_866993.html>

19 CALDERONE Michael, "Huffington Post Awarded Pulitzer Prize", huffingtonpost.com, April 16, 2012, available at: <http://www.huffingtonpost.com/2012/04/16/huffington-post-pulitzerprize-2012_n_1429169.html>

20 HUFFINGTON Arianna, "HuffPost Live: We Need You!", huffingtonpost.com, July 10, 2012, available at: <http://www.huffingtonpost.com/arianna-huffington/huffpost-live-we-needyou_b_1657824.html>

21 SHEA Danny, "HuffPost Live Launches: Huffington Post Debuts Streaming Network", huffingtonpost.com, July 10 2012, available at: <http://www.huffingtonpost.com/2012/08/12/ huffpost-live-launches-hu_n_1771151.html>

22 ELLIS Justin, "Live broadcast: Why The Huffington Post and Boston.com are getting into streaming media", NienmanLab, August 13, 2012, available at: <http://www.niemanlab. org/2012/08/live-broadcast-why-the-huffington-post-and-boston-com-are-getting-intostreaming-media/>

23 ELLIS Justin, " The aggregator builds a magazine: The Huffington Post slows itself down with Huffington", NiemanLab, June 14, 2012, available at: <http://www.niemanlab.org/2012/06/theaggregator-builds-a-magazine-the-huffington-post-slows-itself-down-with-huffington/>

24 HUFFINGTON Arianna, "Covering the World: Introducing The WorldPost", huffingtonpost. com, January 1, 2014, available at: <http://www.huffingtonpost.com/arianna-huffington/ 
Cette dernière phrase apparaît pour la première fois lors de la mise en ligne du site en 2005 : "Le Huffington Post offre le meilleur de l'ancienne et de la nouvelle garde », explique sa directrice à la presse ${ }^{25}$, puis en 2011 lors du lancement de l'édition britannique « Nous mettrons en avant ce que les nouveautés offrent de mieux, l'immédiateté, la transparence et l'interactivité, tout en conservant le mieux de l'ancien: la vérification des faits, l'exactitude, l'équité, et l'accent mis sur la narration ${ }^{26}$, puis de l'édition française en 2012 : «Nous associerons les fondements $d u$ journalisme traditionnel - la poursuite de la vérité, l'exactitude et l'équité - au meilleur de ce que les nouvelles technologies ont à offrir: l'immédiateté, la transparence et l'engagement. ${ }^{27}$ Elle témoigne de la volonté des gestionnaires du site d'associer les pratiques rendues possibles grâce au web 2.0 et à celles qui forment le socle indiscutable du journalisme, dans le but de légitimer leur propre offre qui oscille entre les deux.

D'ailleurs, si un tel flottement est rendu possible, c'est grâce à deux éléments : d'abord, le caractère fondamentalement hybride du média qui n'est pas uniquement un agrégateur de blog, ni exclusivement un producteur d'informations originales. Il est les deux à la fois et combine le contrôle lâche de certaines productions (essentiellement les billets de blogs) à une administration plus stricte d'autres. Ensuite, le déplacement du focus du média en lui-même vers le journalisme, dont le rôle social est censé transcender les divergences entre les 4 pôles de l'énonciation.

Ce que cette position implique, c'est un nivellement entre les productions professionnelles et anonymes, une égalisation, tant au niveau de la pratique que du rendu, des publications proposées par les journalistes du Huffington Post et celles offertes par les lecteurs; en somme la réduction du journalisme à l'acte de discourir sur l'actualité de manière continuelle et, si possible, virale.

covering-the-world-introducing-the-worldpost_b_4637990.html>

25 "Daily Content From Arianna Huffington's Groundbreaking New Huffington Post Available to Newspapers From Tribune Media Services", Tribune Media Services, April 26, 2005, available at: <http://www.prnewswire.com/news-releases/daily-content-from-arianna-huffingtonsgroundbreaking-new-huffington-post-available-to-newspapers-from-tribune-mediaservices-54412112.html>

26 HUFFINGTON Arianna, "Introducing HuffPost UK... And Why We're Excited to Be Joining Your Thriving, Innovative Media Culture", huffingtonpost.com, April 9, 2011, available at: <http:// www.huffingtonpost.co.uk/arianna-huffington/introducing-huffpost-uk_b_890636.html> 27 HUFFINGTON Arianna, «Bienvenue sur Le Huffington Post! », huffingtonpost.fr, 22 janvier 2012, disponible à l'adresse suivante : <http://www.huffingtonpost.fr/arianna-huffington/ bienvenue-sur-le-huffington-post_b_1222277.html> 


\section{Conclusion}

Si la position du Huffington Post était minoritaire dans la sphère médiatique américaine en 2005, elle s'est depuis normalisée à la faveur de l'éclatement progressif des frontières du journalisme (LE CAM \& RUELLAN, 2014) et de l'attractivité de l'offre éditoriale du site. Révélatrice du positionnement périphérique du pure player dans le système journalistique du début des années 2000, elle est devenue, rétrospectivement, le symbole de la clairvoyance d'Arianna Huffington et de ses collaborateurs dont le discours a su accompagner les évolutions du journalisme à l'heure du numérique. Sa pertinence, commerciale tout du moins, s'est d'ailleurs vu confirmée avec l'apparition d'autres médias hybrides, à mi-chemin entre le journalisme et le divertissement, dont BuzzFeed qui, depuis 2014, multiplie les incursions dans le monde de l'information « sérieuse " tout en conservant son modèle éditorial basé sur le divertissement viral ${ }^{28}$.

Leur succès et plus encore leur communication institutionnelle qui acquiert au fil des ans un caractère aussi bien hégémonique qu'universaliste gagneraient à être étudiés plus amplement. La prochaine étape de la recherche sur le discours positionnel du Huffington Post pourrait, ainsi, interroger la validité d'une telle narration mobile dans des contextes nationaux variés, dont la France, le Canada, le Brésil et le Monde Arabe et d'évaluer l'impact des positions « décentrées » sur la communication officielle du groupe.

\section{Références bibliographiques}

\section{CHARAUDEAU Patrick, Le discours d'information médiatique. La} construction du miroir social. Paris : Nathan / Institut national de l'audiovisuel (coll. « Médias-Recherches »), 1997

DEUZE Marc, The Web and its Journalisms: Considering the Consequences of Different Types of News Media Online, New Media \& Society 5 (2), 2003, pp.203-230, available at: <http://www.multidesign. org/lib/Mark+Deuze+-+The+web+and+its+journalisms.pdf $>$

28 RIEDER Rem, "BuzzFeed a burgeoning journalistic force”, USAToday.com, June 16 2015, available at: <http://www.usatoday.com/story/money/columnist/rieder/2015/06/15/ buzzfeed-expands-as-journalism-force/71250444/> 
JOST François, Comprendre la télévision et ses programmes. Paris : Armand Colin, 2e Édition [2005], 2009

LE CAM Florence \& RUELLAN Denis (dir.) Changements et permanences du journalisme. Paris: L'Harmattan, 2014

MARTIKAINEN Maarit, Towards Dialogical Online Journalism, University of Tampere Research Report, 2000, available at: <http:// mansetori.uta.fi/report/ martikainen.pdf> 